\title{
Application of Multi-Agent Theory in Social Networks
}

\author{
Jian Zhou ${ }^{1, a}$, Ning Cai ${ }^{1, b^{*}}$ and Yanjun $\mathrm{Li}^{2, \mathrm{c}}$ \\ ${ }^{1}$ College of Electrical Engineering, Northwest University for Nationalities, China \\ ${ }^{2}$ School of Economics, Northwest University for Nationalities, China

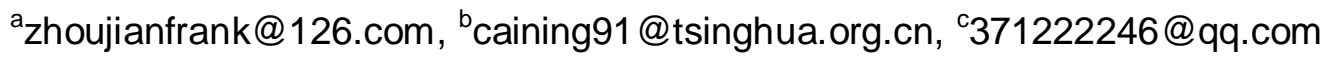 \\ ${ }^{*}$ The corresponding author
}

\begin{abstract}
Keywords: Multi-Agent; Social economics; Complex network; Leader node
\end{abstract}
\begin{abstract}
This paper discusses the dynamic multi-agent system is scattered by the configuration of many autonomous or semi-autonomous subsystem (agent) through the information network which is formed by the interconnection system. Individuals with relatively equal status in the system and each other for information exchange, coordinated movement. Group behavior is a common phenomenon in the nature, the whole group can in macro reflect a more complicated and more efficient than individual behavior. In engineering applications, some tasks cannot be achieved by a single system and needs to be done many subsystems together, such as uav reconnaissance, distributed collaborative filtering and observation, robot soccer game, sensor network data fusion, etc. Study of dynamic multi-agent system is the expansion of traditional control theory, on the traditional isolation system are already mature some basic concepts of system analysis and comprehensive, such as stability, balance, controllability, servo controller, etc., has a new meaning and new problems. In addition, in many industries and has a broad application prospect, such as economic system, the oil refining system, power system, transport system, process system and military system, etc. As a result, nearly five years related research has become the international leading one of the most active direction of the control theory.
\end{abstract}

\section{Introduction}

Controllability disposable reflect system input to the system movement, is an important concept in system analysis. Group of controllability problem is the core of network controllability. In the network, a node as the leader, the node for followers, controllability characterization of leader, network node dominant status in the network movement. In the field of control, this is a new problem, and the traditional isolation system controllability have distinction. Since 2005, there have been some about dynamic multi-agent system controllability analysis work. Tanner [1] for the first time based on the Laplacian matrix of partitioned according to give the definition of network controllability, this definition has been widely recognized by academic circles. Around the stability of dynamic multi-agent system, theoretical study is one of the most group consistency problem, namely the group state asymptotically converge. Group consistency is actually a group of stability is a special form of [2], the global asymptotic stability. In $2004 \sim 2004$, a few papers of founding [3,4], this paper mainly discusses the first-order multi-agent system consistent criterion of groups. Beginning in 2007, there have been some of high order dynamic multi-agent system consistency criterion results [5,6]. In recent years, group consistency analysis about linear time-invariant system theory has been relatively mature. High order system [7,8] consistency problem is equivalent to more than low order system stabilization problem at the same time, it has become the consensus of academia. However, for time-varying, nonlinear and the group of stability of the system [10] with time delay and uncertainty problem has broad space exploration [11]. This paper is based on the research of the early successes, promote the study of group of stability theory.

\section{Model Formulation}

Step1: Based on dynamic multi-agent system of urban and rural labor transfer model, and can 
effectively analysis and forecasting.

Step2: In an arbitrary directed weighted complex network is almost based on the decoupling, prove necessary and sufficient condition with time-varying network topology of system stability, almost yan controllability of complex networks, and simplify the Bauer in matrix theory -- Fike theorem.

Step3: Gives a method based on adaptive leaves the system cluster synchronization conditions, this method does not need to master the characteristics of any global information.

Step4: The consistency of different types of inductive groups. In view of the higher order linear time-invariant systems, criterion conditions are determined for each type.

Step5: For a Leader-followers model of dynamic multi-agent system, put forward a kind of generalized multivariate controllability standard.

Step6: Put forward a kind of network clustering algorithm based on group's consistency and applied to social network node classification of community.

For systems with time-varying network topology stability problem, we will first find an approximate system to replace the original system, the system is equivalent with the original system of stability, and the network topology of the system can be decoupled. This approximate system for nonsingular linear transformation, can be converted into a series of isolated and unrelated low dimensional systems. After the network topology completely equivalent decoupling, breaking the time-varying system stability the limitation of the method of spectral analysis cannot be applied, can be based on Hurwitz criterion of stability conditions are obtained. For lu system cluster synchronization issues, we will use the adaptive method, under the condition of the global information does not have any cluster synchronization. Lu in the system is the essence of the simplest nonlinear system, is a typical significance.

\section{Controllability Problem Analysis}

We proposed a dynamical complex network model, which has several unlinear nodes. Consequently, we can assume that study any directed weighted network of consistency and research the controllability problem as follows:

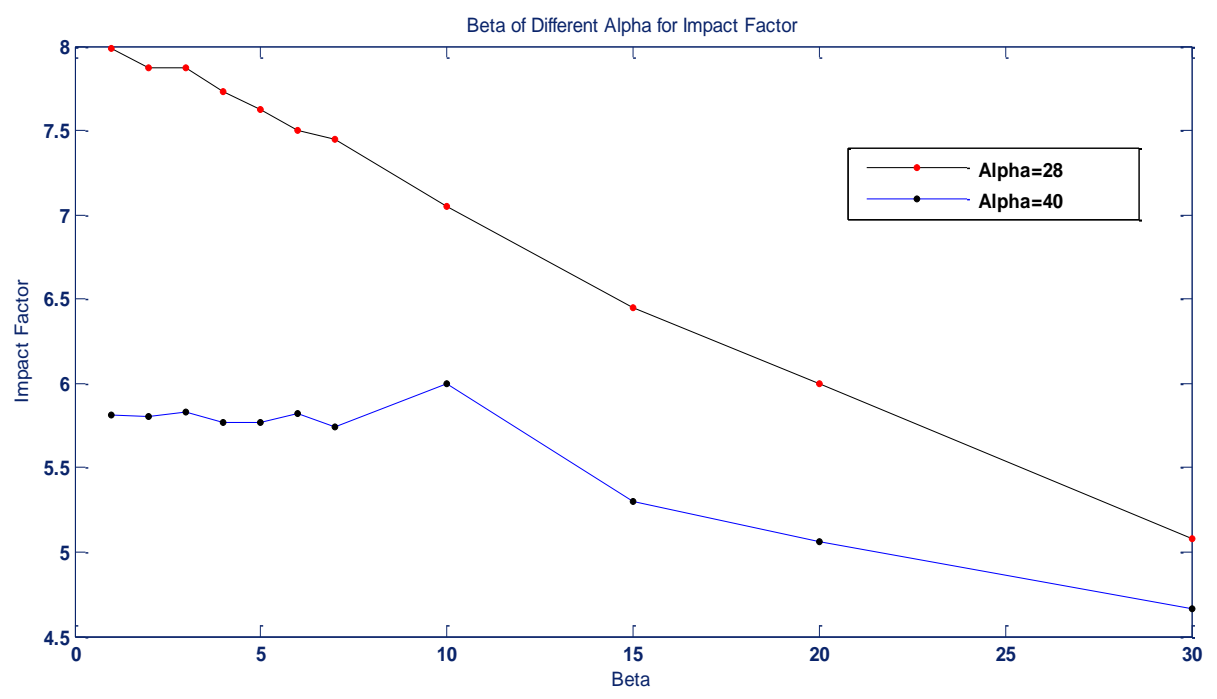

Figure 1. Beta of Different Alpha for the Impact of Network

The main contribution is elaborating that for real-world complex networks unless there is precise ideal modeling, almost every network is controllable in the sense of Kalman controllability, and any infinitesimal perturbation could lead to a controllable network. Discussions around almost controllability will be addressed on two concrete algebraic representations of complex networks respectively: adjacency matrix and Laplacian matrix. The analysis will be conducted both 
theoretically and empirically, or in another word, in mathematical and physical manners.

The dynamic motion of the first type is described as follows:

$$
\begin{aligned}
& x=\frac{d x(t)}{d t}=a x+u \\
& y=\frac{d y(t)}{d t}=a y+u
\end{aligned}
$$

The dynamic motion of the second type of dynamical complex network is described as follows:

$$
\begin{aligned}
& x_{i}=\frac{d x_{i}(t)}{d t}=\sum_{j=1}^{N} a_{i j}\left(x_{j}-x_{i}\right)+u_{i} \\
& y_{i}=\frac{d y_{i}(t)}{d t}=\sum_{j=1}^{N} a_{i j}\left(x_{i}-x_{j}\right)+u_{j}
\end{aligned}
$$

Through the above analysis of the stability of the economic system can drawn by computer simulation waveform diagram as shown below:

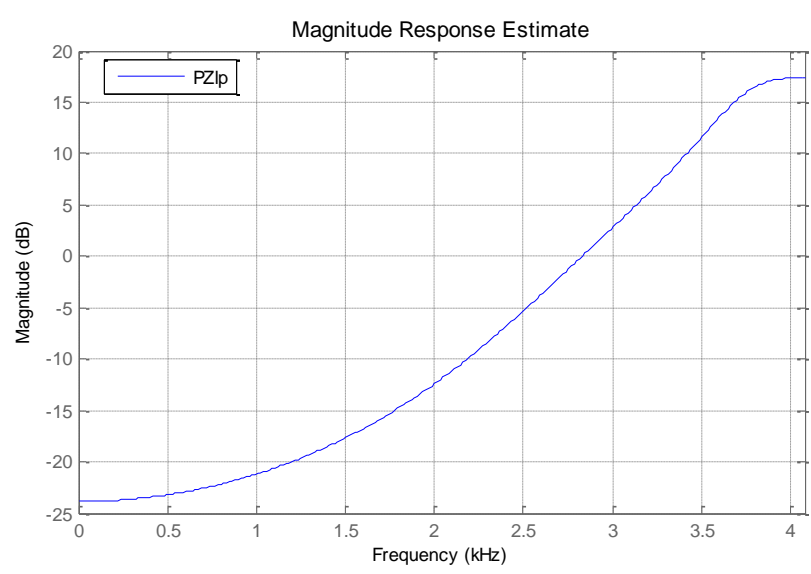

Figure 2. Analysis of Signals Train Vector

It is easily to find that if a network has 3 nodes which is controability and stability. In fact, it is known by the control theory community that almost any given linear system is controllable, i.e. the probability for any system to be exactly controllable.

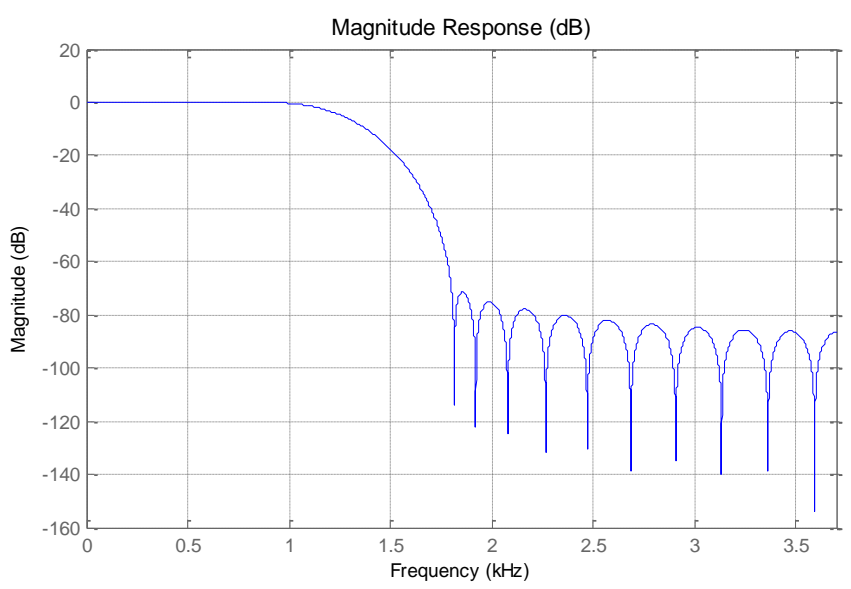

Figure 3. Analysis of Spectra Chirpse 


\section{Conclusion}

This paper introduces a novel approach of clustering and approach of a complex economics network which is based on group consensus of dynamic linear high-order multi-agent systems. The graph topology is associated with a selected multi-agent system, with each agent corresponding to one vertex. In order to reveal the cluster structure, the agents belonging to a similar cluster are expected to aggregate together. As theoretical foundation, a necessary and sufficient condition is given to check the group consensus. Two numerical instances are shown to illustrate the process of approach.

\section{References}

[1] N. Cai, J. Xi, Y. Zhong, \& H.-Y. Ma, "Controllability improvement for linear time-invariant dynamical multi-agent systems”, Int. J. Innov. Comput. I., vol. 8, pp. 3315-3328, 2012.

[2] B. Liu, T. Chu, \& L. Wang et al., "Controllability of a leader-follower dynamic network with switching topology”, IEEE Trans. Autom. Control, vol. 53, pp. 1009-1013, 2008.

[3] Z. Ji, Z. Wang, \& H. Lin et al., "Interconnection topologies for multi-agent coordination under leader-follower framework", Automatica, vol. 45, pp. 2857-2863, 2009.

[4] Z. Yuan, C. Zhao, \& Z. Di et al., "Exact controllability of complex networks", Nat. Commn., vol. 4, 2013.

[5] Cai N., Xi J.-X., Zhong Y.-S., "Swarm stability of high order linear time-invariant swarm systems", IET Control Theory Appl., vol. 5, pp. 402-408, 2011.

[6] Ren W., Beard R. W., "Consensus seeking in multiagent systems under dynamically changing interaction topologies”, IEEE Trans. Automat. Control, vol. 50, pp. 655-661, 2005.

[7] Lin P., Jia Y., Li L., "Distributed robust H-infinity consensus control in directed networks of agents with time-delay", Syst. Control Lett., vol. 57, pp. 643-653, 2008.

[8] Li Z., Duan Z., Chen G., et al., "Consensus of multi-agent systems and synchronization of complex networks: A unified viewpoint”, IEEE Trans. Circuit Syst.-I, vol. 57, pp. 213-224, 2010.

[9] D. B. Payne and J. R. Stern, "Wavelength-switched pas- sively coupled single-mode optical network," in Proc. IOOC-ECOC, 1985, pp. 585-590.

[10]D. Ebehard and E. Voges, "Digital single sideband detection for interferometric sensors," presented at the 2nd Int. Conf. Optical Fiber Sensors, Stuttgart, Germany, Jan. 2-5, 1984.

[11]H.G. Tanner, "On the controllability of nearest neighbor interconnections", Proc. IEEE Conf. Decision and Control, vol. 3, pp. 2467-2472, 2004. 